\title{
Infectious recombinant Senecavirus A expressing novel reporter proteins
}

\author{
Minmin Wang ${ }^{1} \cdot$ Chunxiao Mou ${ }^{1} \cdot$ Mi Chen ${ }^{1} \cdot$ Zhenhai Chen $^{1,2,3}$ (I) \\ Received: 7 December 2020 / Revised: 2 February 2021 / Accepted: 14 February 2021 / Published online: 3 March 2021 \\ (C) The Author(s), under exclusive licence to Springer-Verlag GmbH, DE part of Springer Nature 2021
}

\begin{abstract}
Senecavirus A (SVA) is an emerging picornavirus that has been associated with vesicular disease and neonatal mortality in swine. The construction of SVA virus carrying foreign reporter gene provides a powerful tool in virus research. However, it is often fraught with rescuing a recombinant picornavirus harboring a foreign gene or maintaining the stability of foreign gene in the virus genome. Here, we successfully generated recombinant SVA GD05/2017 viruses (V-GD05-clone) expressing the green fluorescent protein (iLOV), red fluorescent protein (RFP), or NanoLuc luciferase (Nluc). These recombinant viruses have comparable growth kinetics to the parental virus. Genetic stability analysis indicated that V-GD05-iLOV was highly stable in retaining iLOV gene for more than 10 passages, while V-GD05-RFP and V-GD05-Nluc lost the foreign genes in five passages. In addition, highintensity fluorescent signals were found in the V-GD05-RFP- and V-GD05-iLOV-infected cells by fluorescence observation and flow cytometry analysis, and the luciferase activity assay could quantitatively monitor the replication of V-GD05-Nluc. In order to identify the porcine cell receptor for SVA, anthrax toxin receptor 1 (ANTXR1) was knocked out or overexpressed in the ST-R cells. The ANTXR1 knock-out cells lost the ability for SVA infection, while overexpression of ANTXR1 significantly increased the cell permissivity. These results confirmed that ANTXR1 was the receptor for SVA to invade porcine cells as reported in the human cells. Overall, this study suggests that these SVA reporter viruses will be useful tools in elucidating virus pathogenesis and developing control measures.
\end{abstract}

\section{Key points}

- We successfully generated SVA viruses expressing the iLOV, RFP, or Nluc.

- The iLOV was genetically stable in the V-GD05-iLOV genome over ten passages.

- ANTXR1 was the receptor for SVA to invade porcine cells.

Keywords Senecavirus A · Reverse genetics $\cdot$ Reporter virus $\cdot$ Porcine ANTXR1

Minmin Wang and Chunxiao Mou contributed equally to this work.

Zhenhai Chen

zhenhai@yzu.edu.cn

1 College of Veterinary Medicine, Yangzhou University, 12 Wen-hui East Road, Yangzhou JS225009, China

2 Joint International Research Laboratory of Agriculture and Agri-Product Safety, the Ministry of Education of China, Yangzhou University, Yangzhou, China

3 Jiangsu Co-Innovation Center for Prevention and Control of Important Animal Infectious Diseases and Zoonoses, Yangzhou University, Yangzhou, China

\section{Introduction}

Senecavirus A (SVA) is the only species in the genus Senecavirus of family Picornaviridae (Adams et al. 2015; Chen et al. 2019). Like all picornaviruses, SVA is a nonenveloped viruses approximately in size $25-30 \mathrm{~nm}$ with icosahedral symmetry (Adams et al. 2015; Venkataraman et al. 2008a; Wang et al. 2020a, 2020b). SVA has a single-stranded, positive-sense RNA genome with approximately 7300 nucleotides (nt) in length, which comprises 5' and 3' untranslated region (UTR) and encodes a single open reading frame (ORF) that is subsequently processed to 12 polypeptides in the standard picornavirus L-4-3-4 layout: leader protein $\left(\mathrm{L}^{\mathrm{pro}}\right)$; the 
four structural proteins VP4 (1A), VP2 (1B), VP3 (1C), and VP1 (1D); the remaining seven non-structural proteins (nsp) 2A, 2B, 2C, 3A, 3B, 3C $\mathrm{C}^{\text {ro }}$, and 3D $\mathrm{D}^{\text {pol }}$ (Chen et al. 2016; Hales et al. 2008). During translation of the SVA polyprotein, primary cleavage is mediated by the $3 \mathrm{C}$ protease, except that ribosomal "skipping" event happens at the $\mathrm{C}$ terminus of 2A, which results in separation of capsid proteins (P1) region from the non-structural protein coding region (Donnelly et al. 2001; Liu et al. 2019; Venkataraman et al. 2008b).

SVA was first discovered from a PER.C6 cell culture in the USA in 2002 (Venkataraman et al. 2008b). Since then, the virus has been developed as an oncolytic agent due to its selective tropism for human tumor cells. Thereafter, SVA has been associated with sporadic cases of vesicular disease in pigs in the USA and Canada (Corner 2012; Pasma et al. 2008). After 2014-2015, outbreaks of SVA-associated vesicular disease (SAVD) have been reported in a number of swine-producing countries around the world (Canning et al. 2016; Guo et al. 2016; Hause et al. 2016; Leme et al. 2016; Montiel et al. 2016; Saporiti et al. 2017; Vannucci et al. 2015; Wu et al. 2016). So far, many aspects of knowledge such as SVA transmission and pathogenesis remain highly lacking. Reverse genetics is a powerful tool to address the issues with developing recombinant reporter virus. Formerly, full-length cDNA infectious clones of SVV-001 (Poirier et al. 2012) and KS15-01 (Chen et al. 2016) harboring the coding sequence of green fluorescent protein (GFP) were constructed, respectively. Unfortunately, foreign genes were unstable in the virus genome through a few passages. Moreover, GFP-tagged SVA was not well suitable for accurate quantification in monitoring the virus replication. Therefore, we initiated the development of novel SVA reporter viruses to deeply characterize the virus life cycle. In the study, we aimed to construct recombinant SVA carrying green fluorescent protein (iLOV), red fluorescent protein (RFP), or Nanoluciferase (Nluc) genes inserted at the junction site between $2 \mathrm{~A}$ and $2 \mathrm{~B}$. To retain the native coding sequences of virus proteins surrounding the foreign gene, we inserted the porcine teschovirus 2A-like (T2A), a stop-restart translational element, at the side of the 2B product within the SVA polyprotein. This study will provide insight into the stability of foreign genes of different sizes in the SVA genome by characterizing these novel reporter viruses and exploit one of their potentials in identifying the cell receptor for SVA infection of porcine cells.

\section{Materials and methods}

\section{Cells and viruses}

BHK-21, HEK293T cells, ST-R cells (IFN- $\alpha / \beta$ receptorknockout ST cell line), and ST-R ANTXR1 KO cells (ANTXR1-knockout ST-R cell line) were cultured in the
DMEM (Gibco, Carlsbad, CA) supplemented with $10 \%$ fetal bovine serum (Sigma-Aldrich, St. Louis, MO) at $37^{\circ} \mathrm{C}$ and $5 \% \mathrm{CO}_{2}$. The wild-type SVA GD05/2017 (GenBank MH316116) strain was isolated and kept at our laboratory (Wang et al. 2019).

\section{Antibodies and reagents}

The SVA-VP3 and -3C mAbs were made by our laboratory. The Myc and GAPDH mAbs were purchased from Sigma (Sigma-Aldrich, St. Louis, MO). The Dylight 488-labeled goat anti-mouse IgG, Dylight 549-labeled goat anti-rabbit IgG, HRP-labeled goat Anti-mouse IgG, and HRP-labeled goat anti-rabbit IgG were purchased from Abbkine (Wuhan, China). Lipofectamine ${ }^{\circledR} 3000$ was purchased from Invitrogen (Shanghai, China). ANTXR1 mAb was purchased from Bioss (Beijing, China).

\section{Construction of SVA full-length cDNA clones}

The full-length SVA cDNA clones were constructed as described previously (Wang et al. 2020a, 2020b). Four separate fragments (named A to D) were amplified from the SVA GD05/2017 cDNA and assembled into pEGFP-C3 as shown in the Fig. 1a. The resulting full-length cDNA clone was designated as pC3-SVA-GD05. To construct recombinant SVA expressing reporter proteins, the iLOV, RFP, and Nluc genes were amplified from the pUC57-Nluc, pTag-RFP, and pUC57-iLOV. The foreign genes fused with a $\mathrm{T} 2 \mathrm{~A}$ at its $\mathrm{C}$ terminus were inserted between the $2 \mathrm{~A}$ and $2 \mathrm{~B}$ of $\mathrm{pC} 3-\mathrm{SVA}-\mathrm{GD} 05$. These recombinant plasmids were sequenced and designated as pC3-SVA-GD05-Nluc-T2A, pC3-SVA-GD05-iLOVT2A, and pC3-SVA-GD05-RFP-T2A (Fig. 1b). Primers were designed based on the conserved genomic regions of multiple SVA strains (Tables S1 and S2).

\section{Recovery of recombinant viruses}

BHK-21 cells were seeded in the 6-well plates and transfected with pC3-SVA-GD05 and its recombinants. Transfection was conducted using Lipofectamine ${ }^{\circledR} 3000$ following the manufacturer's instructions. At $72-\mathrm{h}$ post-transfection, cell culture supernatants from the BHK-21 cells were transferred to the ST-R cells. Cytopathic effect (CPE) was monitored daily after infection. The recombinant virus recovered from pC3-SVA-GD05 was designated as V-GD05-clone. The same method was used to rescue the iLOV, RFP, and Nluc-reporter viruses (VGD05-iLOV, V-GD05-RFP, and V-GD05-Nluc). The viral protein expression in the recombinant virus-infected cells was detected by fluorescence observation, western blot, luciferase activity assay, and flow cytometry. 


\section{Genetic stability of reporter viruses in the ST-R cells}

Reporter viruses had been serially cultured from the first passage (P1) to the tenth passage (P10) in the ST-R cells. The viral RNAs of P3, P5, and P10 virus stocks were extracted and reverse transcribed into cDNA. The foreign gene regions were examined by PCR and sequence analysis.

\section{The growth curve of recombinant viruses}

ST-R cells were infected with P3 recombinant viruses at an MOI of 0.1 . The culture supernatants were collected at 6,12 , $24,36,48$, and 60 hpi, respectively. Virus titer was determined by CPE observation, and quantified as $50 \%$ tissue culture infective dose $\left(\mathrm{TCID}_{50}\right) \mathrm{mL}^{-1}$ according to the ReedMuench method (Reed and Muench 1938).

\section{Immunofluorescence assay}

The ST-R cell monolayers were fixed with $4 \%$ paraformaldehyde in PBS (pH 7.4) for 10 min and then permeabilized with $0.1 \%$ TritonX-100 and 2\% BSA in PBS for $30 \mathrm{~min}$ at room temperature. After 1-h incubation with primary antibodies, cell monolayers were washed with PBS for three times and further incubated for $1 \mathrm{~h}$ with Dylight 488 anti-rabbit IgG. The cell nuclei were stained with 4, 6-diamidino-2-phenylindoledihydrochloride (DAPI) performed as suggested by the manufacturer (Molecular Probes). After three times washing by PBS, cells were analyzed under a fluorescent microscope, and pictures were taken with Leica Cell Imaging System (Leica, Germany).

\section{Western blot analysis}

The ST-R cells were treated with lysis buffer (Beyotime Biotech, Shanghai, China). The cell lysate supernatant was mixed with $5 \times$ loading buffer containing $5 \% \beta$ mercaptoethanol and denatured at $100{ }^{\circ} \mathrm{C}$ for $10 \mathrm{~min}$. Proteins were separated on a $12 \%$ SDS-PAGE gel and blotted onto nitrocellulose membrane. The membranes were blocked with 5\% skim milk in the Tris-buffered saline (TBS) containing Tween 20 at room temperature for $2 \mathrm{~h}$. The membranes were incubated with primary antibodies at $4{ }^{\circ} \mathrm{C}$ overnight. After three times washing with TBST, the membranes were incubated by HRPconjugated secondary antibodies. At last, the target proteins were visualized using the chemiluminescence imaging system (Tanon, Shanghai, China).

\section{Flow cytometry analysis}

The SVA-infected ST-R cells were harvested by treatment of $0.25 \%$ trypsin and then washed twice with PBS at $1000 \mathrm{rpm}$ for $5 \mathrm{~min}$, following by suspending cells in the PBS. The specific fluorescence of iLOV and RFP was measured upon excitation at 488 and $561 \mathrm{~nm}$, respectively. iLOV fluorescence was analyzed through FL1 channel and RFP fluorescence was analyzed through FL2 channel. Data from 10,000

a

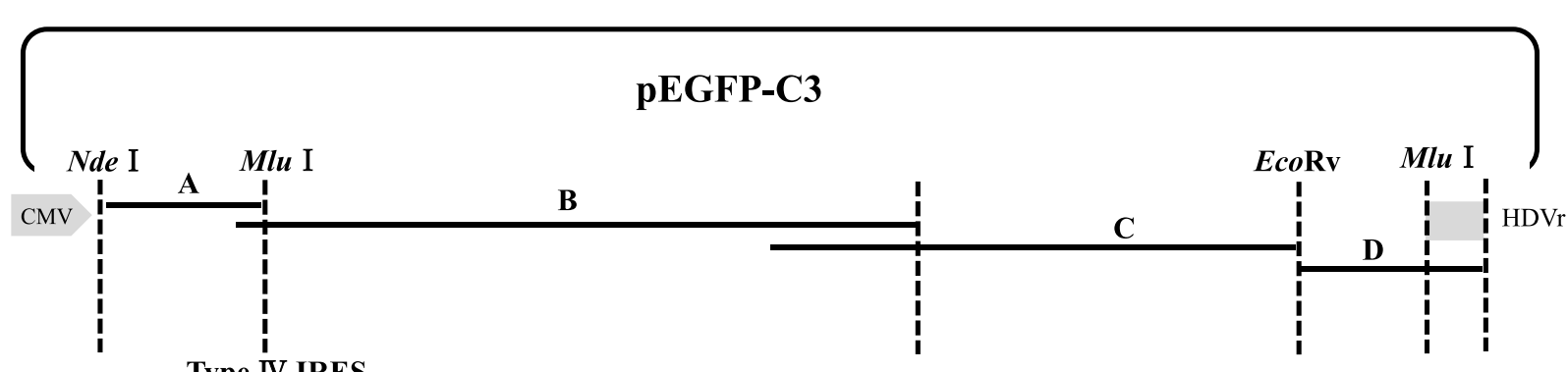

5'UTR

Type IV IRES

3'UTR

b

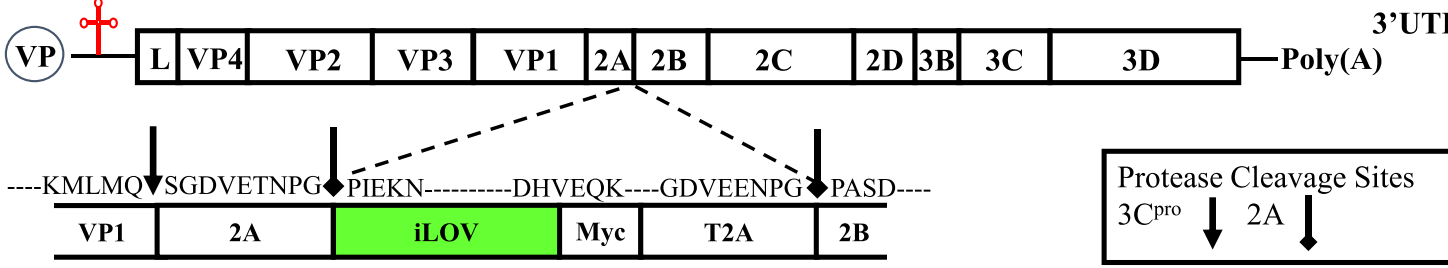

\begin{tabular}{c|c|c|c|c|c|c}
\hline VP1 & 2A & Nluc & Myc & \multicolumn{2}{|c|}{ T2A } & 2B \\
\hline VP1 & 2A & RFP & Myc & T2A & 2B \\
\hline
\end{tabular}

Fig. 1 The schematic diagram for the construction of SVA. a Schematic diagram of the full-length SVA GD05/2017 genome and construction of the full-length cDNA clone. $\mathbf{b}$ Schematic presentation of the insertion of
iLOV, Nluc, and RFP into GD05/2017 genome. Cleavage of polypeptide by $3 \mathrm{C}$ was indicated by arrowhead, while ribosome skipping was indicated by diamond head 
events were recorded, and the data was analyzed using CytExpert Software 2.3 (Beckman Coulter, USA).

\section{Luciferase activity assay}

ST-R cells in the 6-well plates were infected with V-GD05Nluc at an MOI of 0.1 . At $12 \mathrm{~h}, 18 \mathrm{~h}$, and $24 \mathrm{~h}$ post infection, Nano-Glo® Luciferase Assay System (Promega, USA) was used to detect the Nluc activity according to the manufacturer's instructions.

\section{Construction and analysis of ST-R ANTXR1 KO cell line}

The sgRNA was designed based on the gene of porcine ANTXR1 through http://crispor.tefor.net/. The sequences of sgRNAs targeting the first exon of ANTXR1 were \#1F: 5'CACCGCTCATCTGCGCCGGGCAAG-3'; \#1R: 5' AAACCTTGCCCGGCGCAGATGAGC-3'; \#2F: 5'CACCGCAGGTCAAATCCCCCGTAGC-3'; \#2R: 5' AAACGCTACGGGGGATTTGACCTGC-3'. To generate lentivirus expressing sgRNA, HEK293T cells were co- a

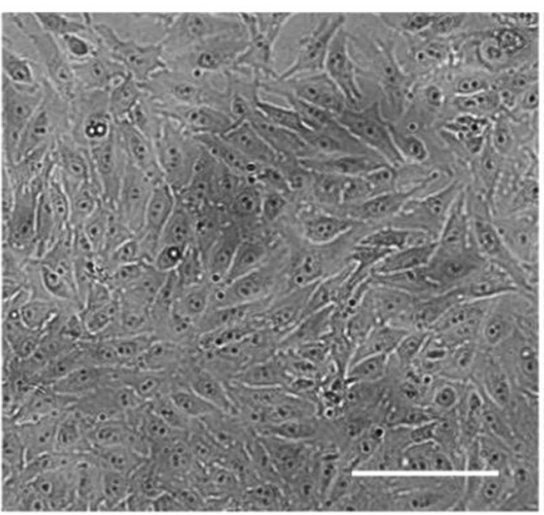

b
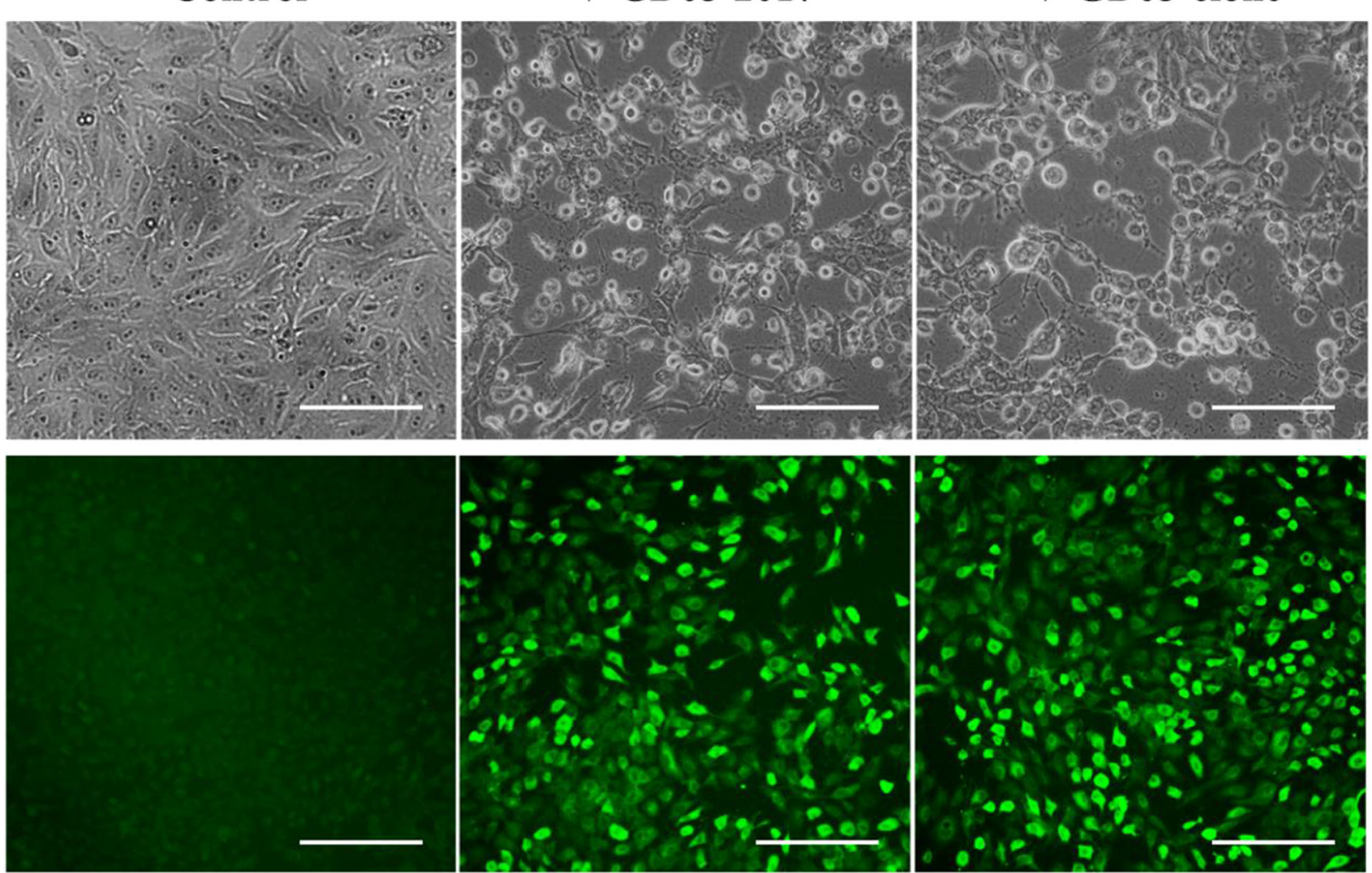

c

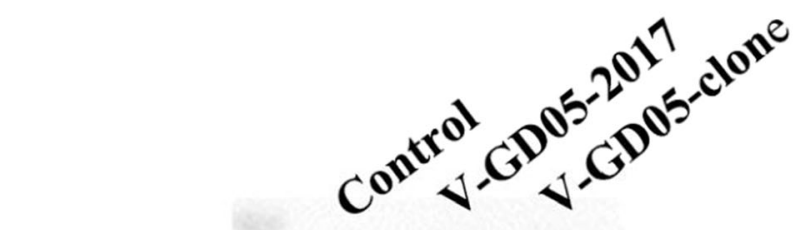

Anti-VP3 d

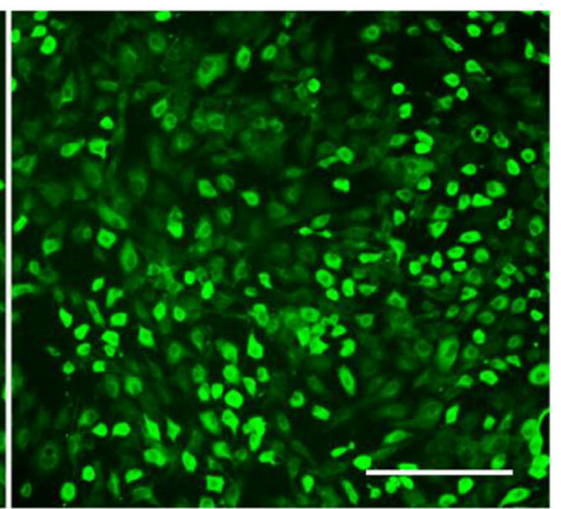

\section{Anti-GAPDH}

Fig. 2 Recovery and identification of recombinant virus V-GD05-clone. a ST-R cells were infected with V-GD05/2017 and V-GD05-clone. After $24 \mathrm{~h}, \mathrm{CPE}$ was observed under microscopy. b Cells were fixed and treated with VP3 mAb followed by addition of Dylight 488 anti-rabbit IgG as

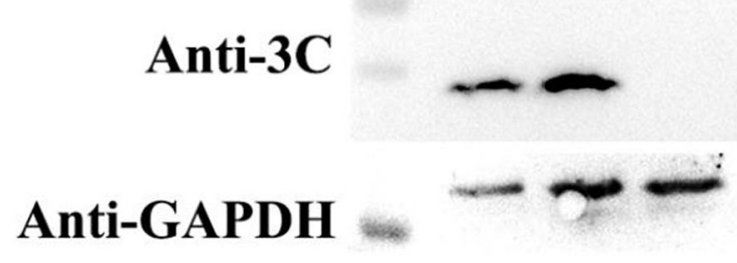

secondary antibody. The bar $=200 \mu \mathrm{m}$. c V-GD05/2017 and V-GD05clone infected cells at an MOI of 0.1 were analyzed by western blot with the VP3 mAb. d V-GD05/2017 and V-GD05-clone infected cells at an MOI of 0.1 were analyzed by western blot with $3 \mathrm{C} \mathrm{mAb}$ 
transfected with Lenti-ANTXR1-sgRNA, packaging vector psPAX2, and envelop vector pMD2.G using Lipofectamine ${ }^{\circledR}$ 3000. Culture supernatants containing lentiviruses were harvested at $24 \mathrm{~h}$ and $48 \mathrm{~h}$ post-transfection and then passed through a $0.45 \mu \mathrm{M}$-pore-size filter. ST-R cells were infected by filtered lentiviruses expressing ANTXR1-sgRNAs to develop ST-R ANTXR1 KO stable cell line. The knockout of ANTXR1 gene was identified by DNA sequencing and western blot.

\section{Overexpression of ANTXR1 in the ST-R cells}

Porcine ANTXR1 gene was amplified from the cDNA of ST$\mathrm{R}$ cells and inserted into pCAGGS-Myc vector. ST-R cells were seeded in the 6-well plates and transfected with pCAGGS-Myc-pig-ANTXR1 (2.5 $\mathrm{g} /$ well). At $24 \mathrm{~h}, 36 \mathrm{~h}$, and $48 \mathrm{~h}$ post-transfection, the cells were infected with $\mathrm{V}$ GD05-RFP, V-GD05-iLOV, and V-GD05-Nluc at an MOI

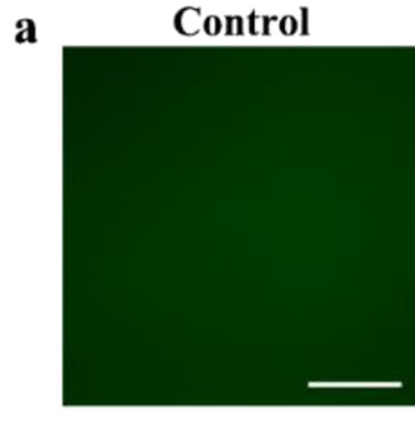

b

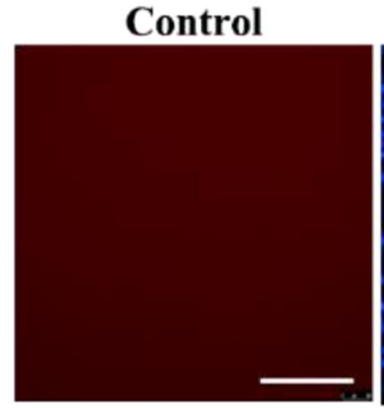

DAPI

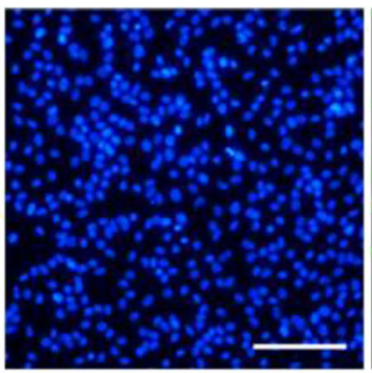

DAPI

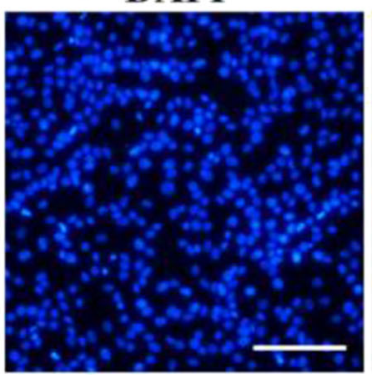

V-GD05-iLOV

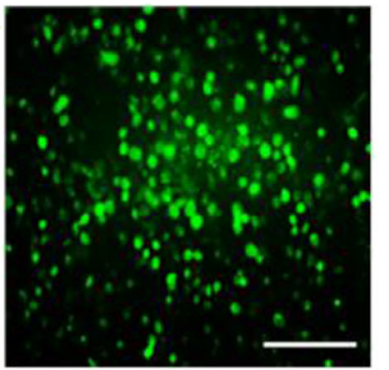

\section{V-GD05-RFP}

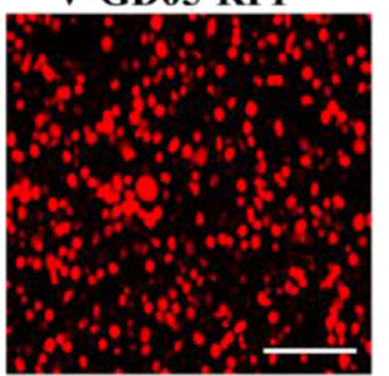

c

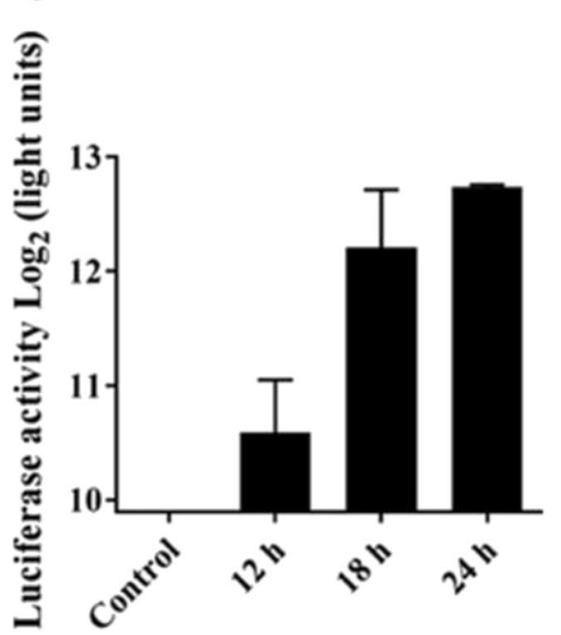

Hours post-infection (hpi)

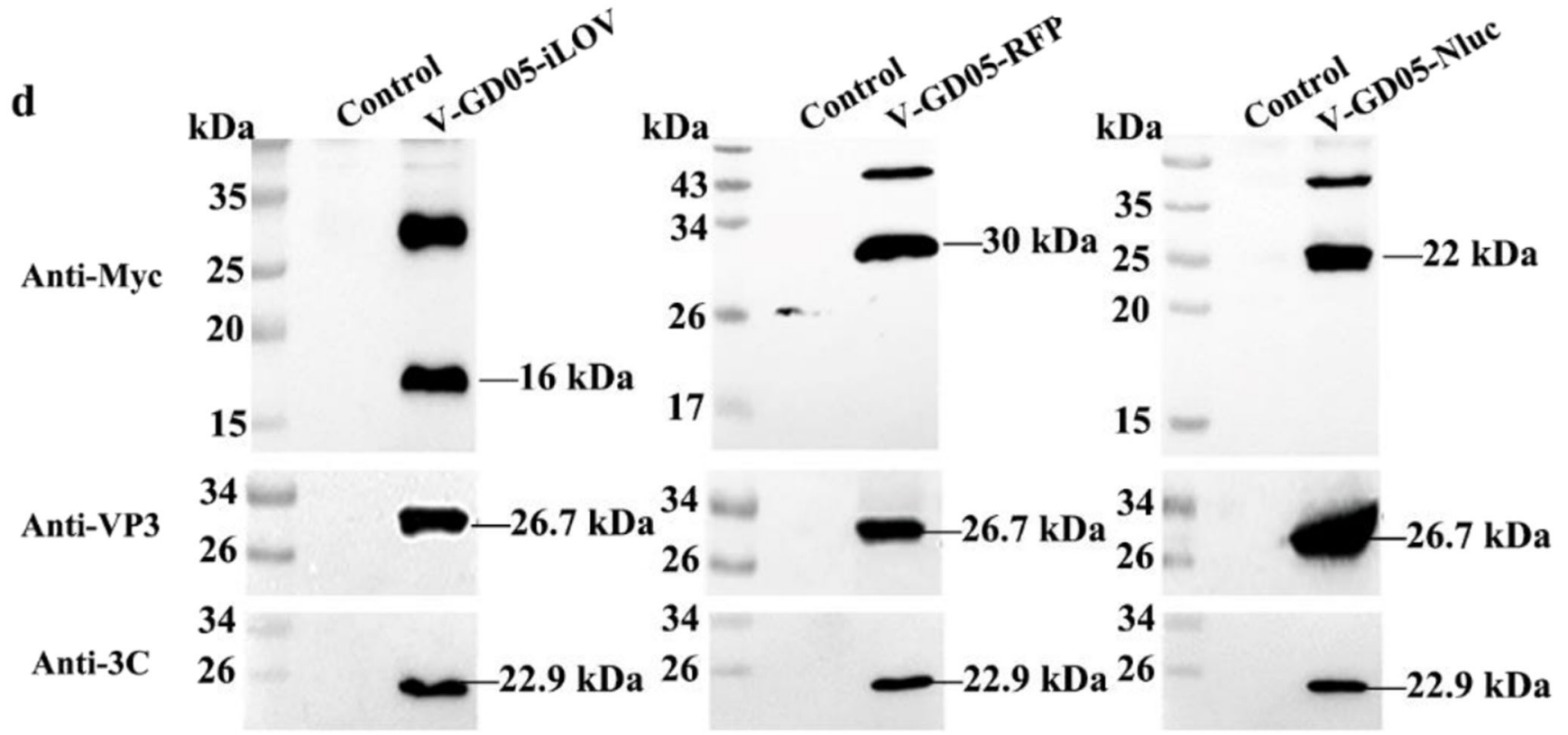

Fig. 3 Characterization of SVA reporter viruses. BHK-21 cells were transfected by pC3-SVA-GD05-iLOV-T2A, pC3-SVA-GD05-RFPT2A. At 3 dpi, cell culture supernatant from the transfected cells was passaged onto ST-R cells. a and b The fluorescence were observed in the V-GD05-iLOV- or V-GD05-RFP-infected cells at 36 hpi. The bar = $200 \mu \mathrm{m}$. c The luciferase activities were detected in the V-GD05-Nluc- infected ST-R cells at 12-24 hpi. d Cell lysates from the reporter viruses infected ST-R cells infected with V-GD05-iLOV, V-GD05-RFP, VGD05-Nluc, or mock-infected cells were harvested at $36 \mathrm{hpi}$. The expression of reporter and viral proteins were analyzed by western blot using myc, VP3, and $3 \mathrm{C}$ mAbs 
of 0.1 for $24 \mathrm{~h}$. The expression of porcine ANTXR1 was detected by western blot.

\section{Identification of ANTXR1 as cell receptor using SVA reporter viruses}

ST-R ANTXR1 KO stable cell lines and porcine ANTXR1overexpressed ST-R cells were used to identify the cell receptor for SVA infection. The ST-R ANTXR1 KO cells were infected with V-GD05-iLOV, V-GD05-RFP, or V-GD05Nluc $(\mathrm{MOI}=0.1)$. At $36 \mathrm{hpi}$, the fluorescence in the $\mathrm{V}$ GD05-iLOV and V-GD05-RFP infected cells was observed by fluorescence microscope. The luciferase activity of $\mathrm{V}$ GD05-Nluc infected cells was measured at 18 hpi by NanoGlo® Luciferase Assay. Additionally, after 24, 36, and $48 \mathrm{~h}$ post-transfection, porcine ANTXR1 overexpressed ST-R cells were infected with V-GD05-RFP, V-GD05-iLOV, or V-
GD05-Nluc (MOI=0.1). At $24 \mathrm{hpi}$, fluorescence and luciferase activities of the infected cells were detected.

\section{Statistical analysis}

Statistical analyses were performed by using one-way analysis of variance (ANOVA) using GraphPad InStat Prism software (version5.0). Statistical significance was expressed as a $P$ value less than 0.05 or 0.01 .

\section{Results}

\section{Construction of SVA infectious clone}

As shown in Fig. 1a, a panel of fragments that span the entire SVA GD05/2017 genome was assembled into pEGFP-C3 to

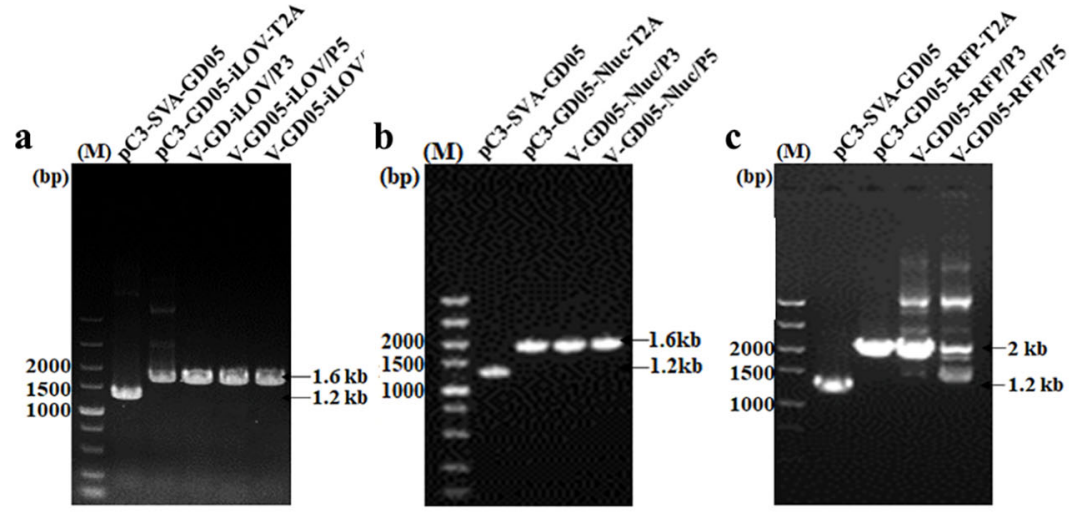

d

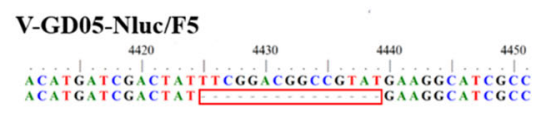

V-GD05-RFP/F5

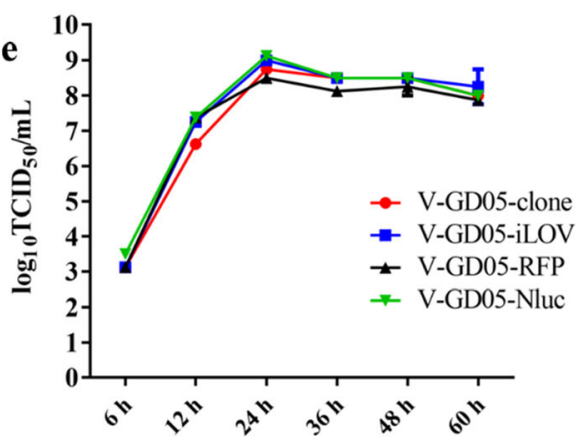

Hours post-infection (hpi)

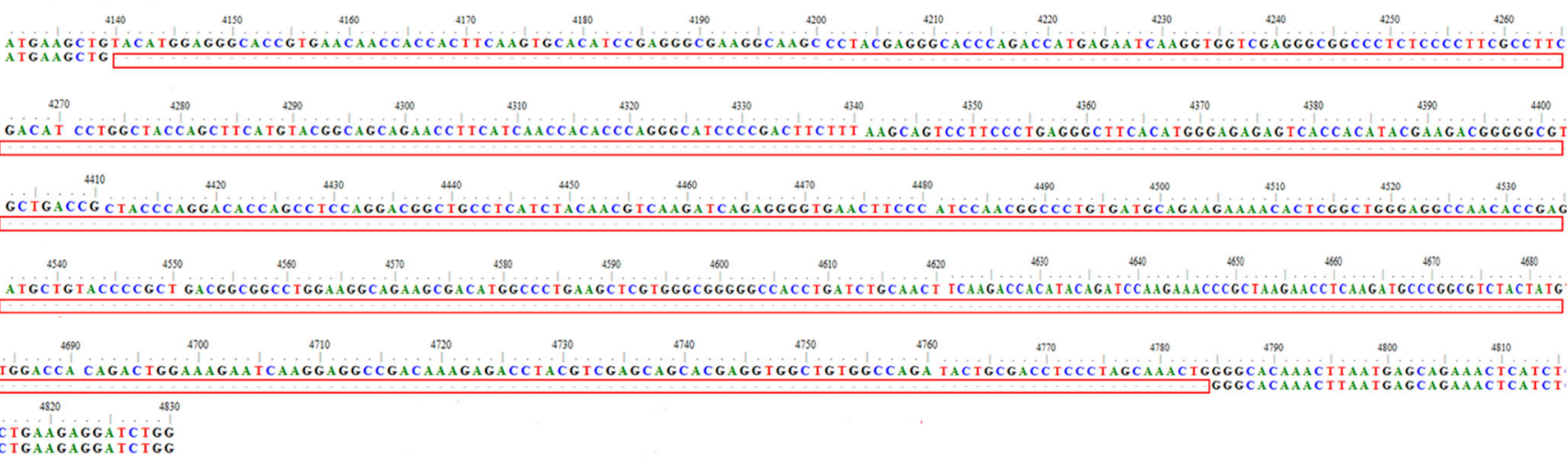

Fig. 4 Genetic stability and growth curve of SVA reporter viruses. a Identification of iLOV gene in the V-GD05-iLOV of P3, P5, and P10 virus stocks with the pC3-SVA-GD05 and pC3-SVA-GD05-iLOV-T2A as controls. b Identification of Nluc gene in the V-GD05-Nluc of P3 and $\mathrm{P} 5$ virus stocks with the $\mathrm{pC} 3-\mathrm{SVA-GD} 05$ and $\mathrm{pC} 3-\mathrm{SVA-GD05-Nluc-}$ $\mathrm{T} 2 \mathrm{~A}$ as controls. $\mathbf{c}$ Identification of RFP gene in the V-GD05-RFP of
P3 and P5 virus stocks with the pC3-SVA-GD05 and pC3-SVA-GD05RFP-T2A as controls. $\mathbf{d}$ Sequencing analysis of the V-GD05-RFP and VGD05-Nluc deletion variants. e ST-R cells were infected with SVA reporter viruses at the MOI of 0.1 and the virus titers in the cell culture supernatants were analyzed at $6,12,24,36,48$, and $60 \mathrm{hpi}$ 
yield pC3-SVA-GD05. In the virus infectious clone, a CMV promoter was placed at the 5 ' terminus of the virus genome, while a polyA tail of 22 residues was inserted at the 3' end. To construct the reporter-tagged SVA viruses, the junction site between the $2 \mathrm{~A}$ and $2 \mathrm{~B}$ was selected for insertion of foreign gene. The iLOV, RFP, and Nluc fused with a T2A peptide were inserted into pEGFP-C3-GD05 (Fig. 1b), and the resulting plasmids were named as pC3-SVA-GD05-iLOV-T2A, pC3-SVAGD05-RFP-T2A, and pC3-SVA-GD05-Nluc-T2A. During the translation of virus polypeptide, the ribosome skips at TNPG $\downarrow P$ of the SVA $2 \mathrm{~A}$ sequence and continues in frame to produce a reporter-T2A fusion protein and then skips a second time at the $\mathrm{T} 2 \mathrm{~A}$ SNPG $\downarrow P$ sequence and continues in frame to translate the remainder of the GD05/2017 polyprotein. The advantage of this strategy is capable of retaining the native $\mathrm{N}$ or $\mathrm{C}$-terminuses of virus proteins surrounding the foreign genes.
The recovery and characterization of recombinant viruses

To rescue the recombinant viruses, $\mathrm{pC} 3$-SVA-GD05, pC3SVA-GD05-iLOV-T2A, pC3-SVA-GD05-RFP-T2A, or pC3-SVA-GD05-Nluc-T2A were transfected into BHK-21 cells. Cell culture supernatants from BHK21 cells were further passaged onto ST-R cells to generate V-GD05-clone, VGD05-iLOV, V-GD05-RFP, and V-GD05-Nluc. In comparison with the parental virus, the V-GD05-clone displayed a similar cytopathic effect (Fig. 2a), and the expression of VP3 could be detected by immunofluorescence (Fig. 2b). Meanwhile, the expression of VP3 and 3C could also be detected by western blot (Fig. 2c and 2d). For characterization of reporter viruses, the iLOV (green) (Fig. 3a) and RFP (red) (Fig. 3b) fluorescence signals were observed in the VGD05-iLOV- and V-GD05-RFP-infected ST-R cells at 36 hpi, while the Nluc luciferase activities were also detectable in the V-GD05-Nluc-infected ST-R cells at 12-24 hpi (Fig. $\mathbf{a}$
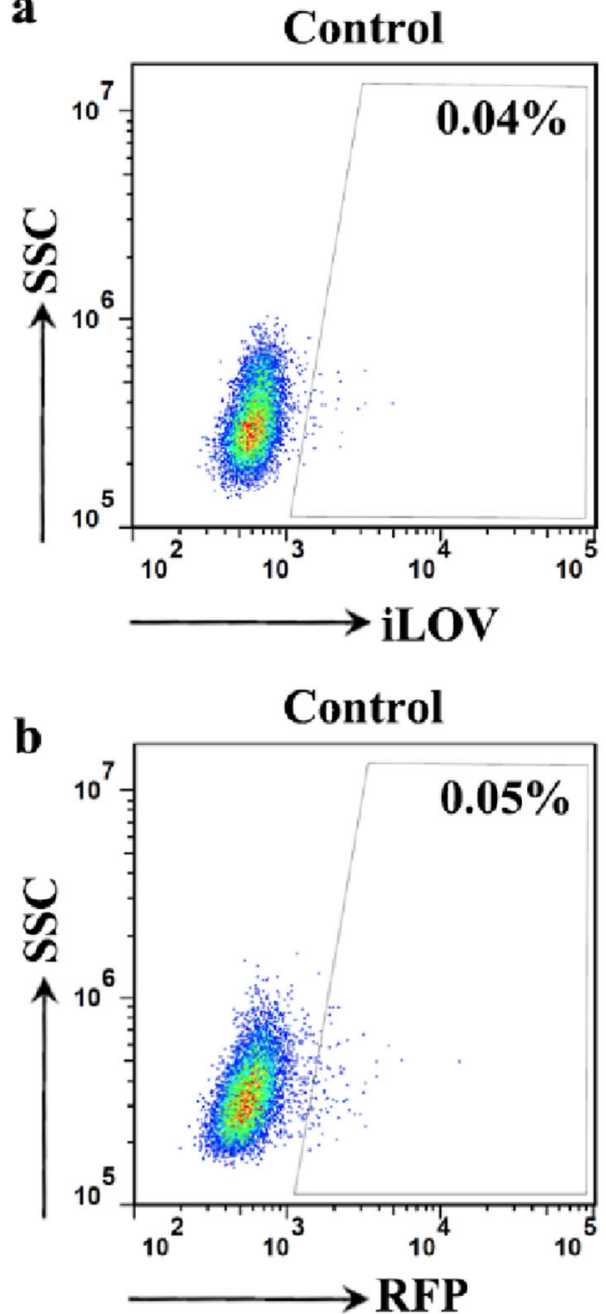

V-GD05-iLOV $12 \mathrm{~h}$
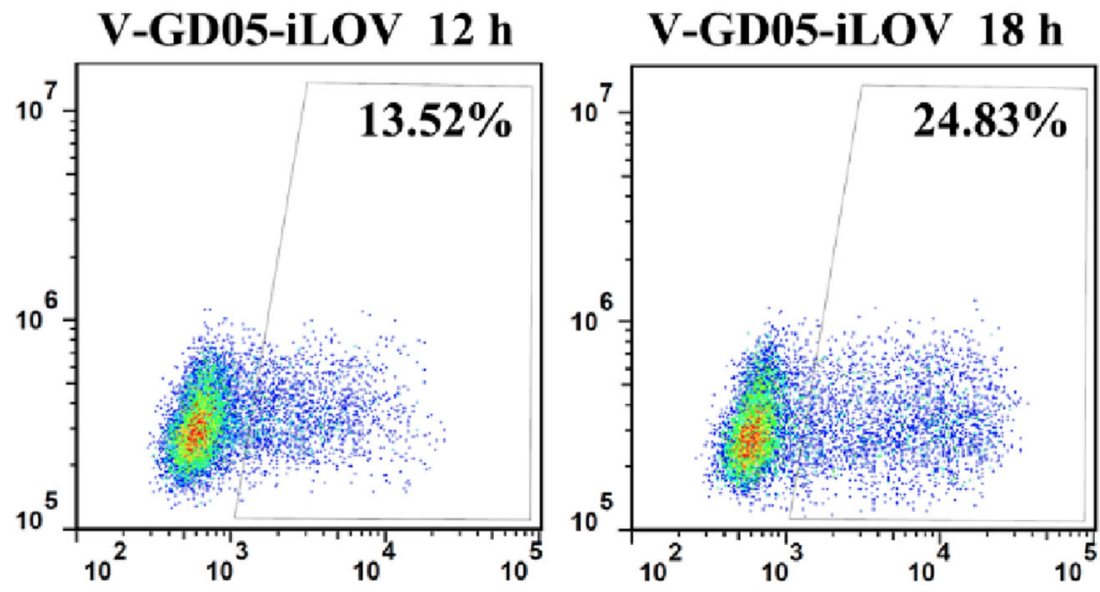

Fig. 5 Detection of SVA reporter virus-infected cells by flow cytometry. ST-R cells were infected with V-GD05-iLOV (a) and V-GD05-RFP (b) at an MOI of 0.1 . After 12 or $18 \mathrm{~h}$, cells were analyzed by flow cytometry 
$3 \mathrm{c}$ ). In the western blot assays (Fig. 3d), the bands of iLOVT2A (16 kDa), RFP-T2A (30 kDa), and Nluc-T2A (22 kDa) were clearly identified, suggesting that the fusion proteins have been processed as expected. However, a larger band was always present in each assay, which may be a partially processed polypeptide containing the reporter proteins. The finding is not unusual in that $2 \mathrm{~A}$ peptide-mediated cleavage efficiency is not $100 \%$.

\section{Genetic stability of reporter viruses}

Picornaviruses are generally limited by genetic instability as an expression vector, and it is therefore necessary to investigate the genetic stability of reporter genes in the SVA genome. Following the initial recovery of rescued viruses in BHK-21, viruses were serially passaged 10 times in the ST-R cells to yield P1 to P10 virus stocks. RT-PCR and sequencing analysis of the P3, P5, and P10 stocks revealed that exogenous RFP and Nluc genes were relatively stable which were retained within the SVA genome over five passages (P1 to P5) (Figs. 4b-d). In comparison, the iLOV gene was consistently intact with no mutations or deletions in the SVA genome through 10 passages (Fig. 4a). Although the results showed that SVA tolerated the iLOV, RFP, and Nluc genes at different degrees, it remained to investigate whether the insertion of additional genes of
300-700 nt into the genome would affect the efficiency of virus replication. Accordingly, virus growth comparisons were performed among V-GD05-clone, V-GD05iLOV, V-GD05-RFP, and V-GD05-Nluc. After infection with recombinant viruses, the ST-R cell culture supernatants were collected at $6,12,24,36,48$, and 60 hpi to measure the virus titer. As shown in Fig. 4e, replication kinetics of the reporter viruses were similar to that of the V-GD05-clone, where all of viruses reached the peak titers at $24 \mathrm{hpi}$. The above results showed the virus replications of V-GD05-iLOV, V-GD05-RFP, and V-GD05-Nluc were not drastically affected in vitro.

The construction of fluorescent or luminescent SVA offers alternative systems to detect SVA-infected cells in vitro and in vivo studies. We next explored flow cytometry by which V-GD05-iLOV and V-GD05-RFP replication can be detected and quantified in cells. As shown in Fig. 5, V-GD05-iLOV and V-GD05-RFP-infected ST-R cells were capable of being detected and quantified using flow cytometry. The numbers of positive ST-R cells expressing fluorescent proteins increased from 13.52 to $24.83 \%$ (V-GD05-iLOV) and from 18.33 to $27.8 \%$ (V-GD05-RFP) at $12 \mathrm{hpi}$ and $18 \mathrm{hpi}$. Moreover, luciferase activities could be detected in the V-GD05-Nluc-infected ST-R cells as high as close to $2^{13}$ comparing to $2^{0}$ in the control cells at $24 \mathrm{hpi}$ (Fig. 3c).

\section{a}

\section{ST-R ANTXR1/wt}

347-GCTC TGGGCATT GGCT TCAAGT TTCTCTC TTTGGCCACT TT TGTGCTCATC TGCGCC GGGCAAGGGGGAC-416

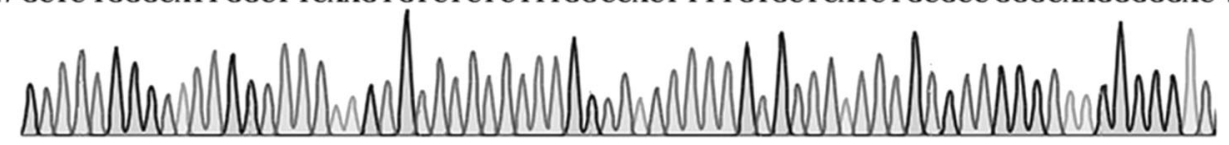

\section{ST-R ANTXR1 KO 1}

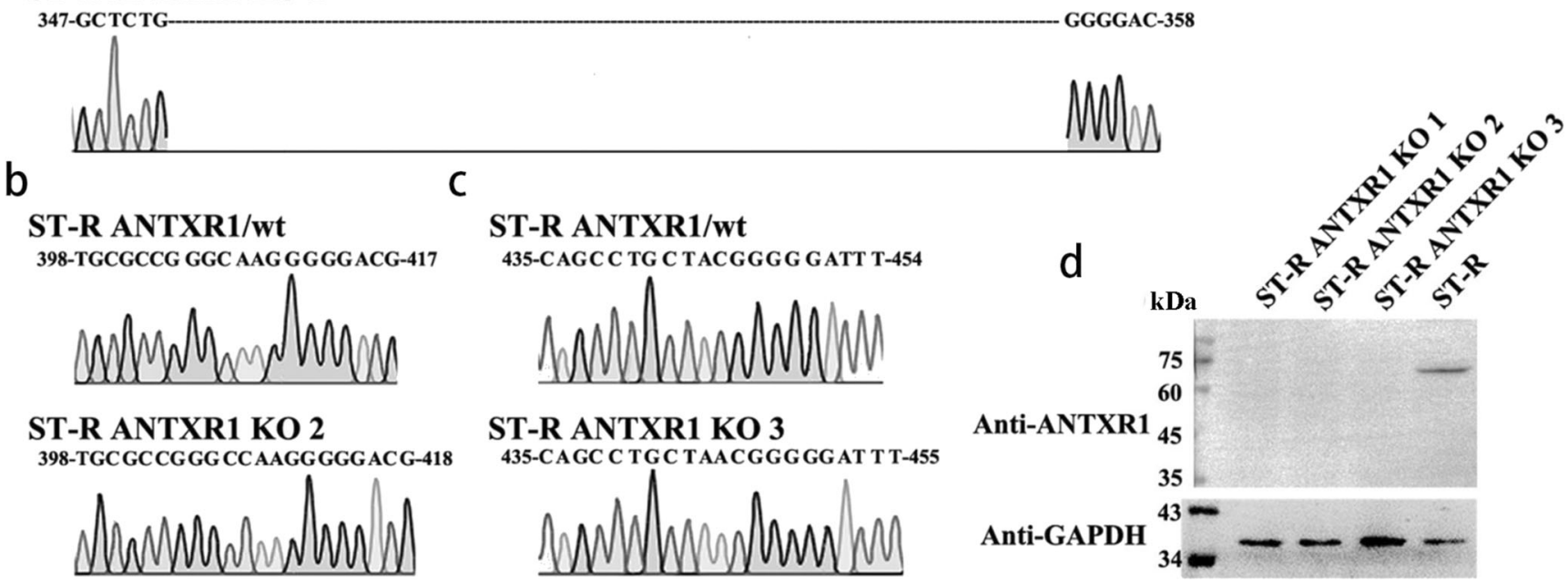

Fig. 6 Identification of porcine ANTXR1 knocked-out cell lines. a Sequencing chromatogram of partial ANTXR1 gene in the ST-R cells and ST-R ANTXR1 KO1 cell line. b Sequencing chromatogram of partial ANTXR1 gene in the ST-R cells and ST-R ANTXR1 KO2 cell line. c
Sequencing chromatogram of partial ANTXR1 gene in the ST-R cells and ST-R ANTXR1 KO3 cell line. $\mathbf{d}$ The expression of ANTXR1 protein in the ST-R and three KO cell lines were detected by western blot using the ANTXR1-specific mAb 


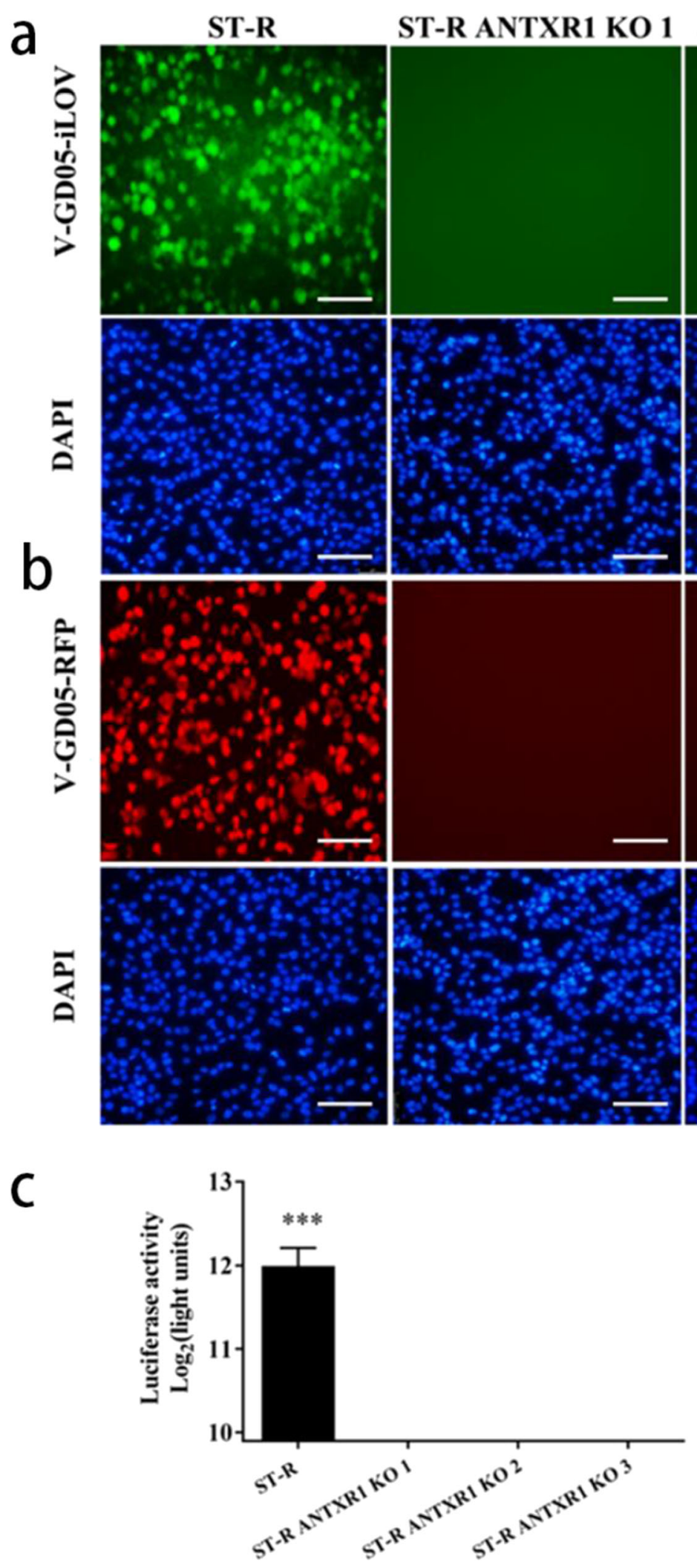

Fig. 7 Identification of ANTXR1 as cell receptor using SVA reporter viruses. ST-R and ST-R ANTXR1 KO cells were infected with VGD05-iLOV(a) and V-GD05-RFP (b) at an MOI of 0.1, and the fluorescence was observed after $36 \mathrm{~h}$ infection. Nuclei were stained blue (DAPI).

The bar $=200 \mu \mathrm{m}$. $\mathbf{c}$ ST-R and ST-R ANTXR1 KO cells were infected with V-GD05-Nluc at an MOI of 0.1. At $18 \mathrm{hpi}$, the luciferase activities were detected 


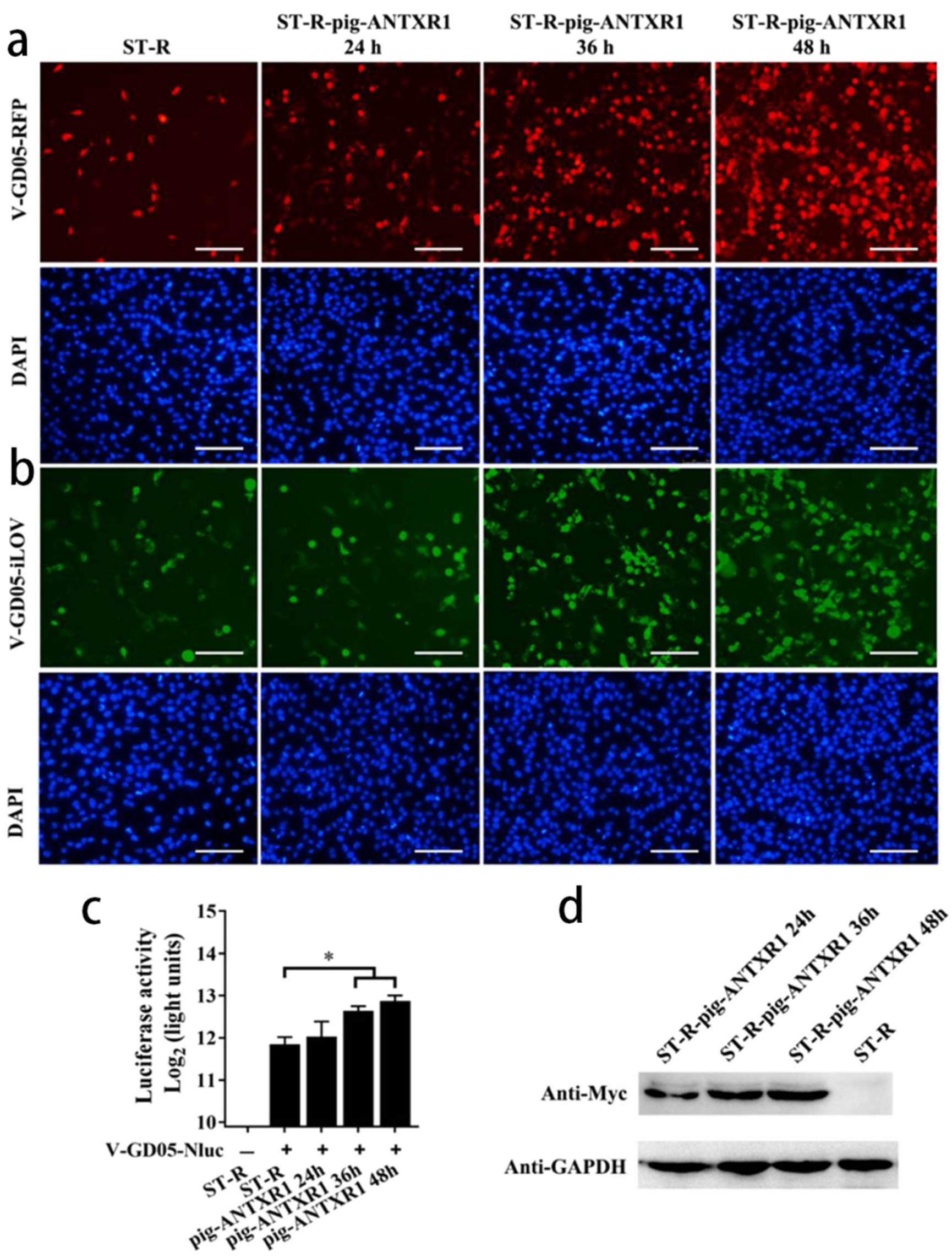


Fig. 8 Overexpression of ANTXR1 increases the cell permissivity for SVA infection. ST-R cells were transfected with pCAGGS-Myc-pigANTXR1. At $24 \mathrm{~h}, 36 \mathrm{~h}$, and $48 \mathrm{~h}$ post-transfection, the cells were infected with V-GD05-RFP (a), V-GD05-iLOV (b), and V-GD05-Nluc (c) at an MOI of 0.1 for $24 \mathrm{~h}$. (d) The expression of myc-tagged porcine ANTXR1 protein were detected through western blot using the mycspecific $\mathrm{mAb}$

\section{Using reporter viruses to identify the cell receptor for SVA infection}

It was reported that ANTXR1 was the cellular receptor for entry of SVV-001 into human cells. To investigate the effect of porcine ANTXR1 for SVA infection, we initially knocked out (KO) the ANTXR1 in the ST-R cells and developed three KO cell lines (ST-R ANTXR1 KO1, KO2, KO3). We sequenced the target region of the genomic DNA extracted from ST-R ANTXR1 KO cell lines to analyze the mutation effect. The sequencing results showed that there was a deletion of 58 nucleotides in the target region of ST-R ANTXR1 KO1 cells (Fig. 6a). We also identified the ST-R ANTXR1 KO2 (Fig. 6b) and ANTXR1 KO3 cells (Fig. 6c), both of which harbored frameshift mutations within the first exon of ANTXR1. Then, the expression of ANTXR1 protein in the ST-R ANTXR1 KO cells was investigated. As expected, porcine ANTXR1 in the ST-R ANTXR1 KO cell lines were unable to detect by western blot using the ANTXR1-specific mAb (Fig. 6d). The ST-R ANTXR1 KO cells were infected with reporter viruses at the MOI of 0.1 . After $36 \mathrm{~h}$, there was no fluorescent signal observed in the ST-R ANTXR1 KO cells infected by V-GD05iLOV (Fig. 7a) and V-GD05-RFP (Fig. 7b), and luciferase activities were also undetectable in the V-GD05-Nlucinfected ST-R ANTXR1 KO cells (Fig. 7c). Furthermore, the porcine ANTXR1 was overexpressed in the ST-R cells transfected by pCAGGS-Myc-pig-ANTXR1. As shown in the Fig. 8d, the expression of Myc-tagged ANTXR1 was identified by western blot. At 24,36 , and $48 \mathrm{~h}$ post-transfection, SVA reporter viruses were then added to infect the ANTXR1overexpressed cells. Compared with ST-R cells, RFP (Fig. 8a), iLOV (Fig. 8b) fluorescence signal and Nluc activities (Fig. 8c) were significantly increased in the ANTXR1overexpressed ST-R cells. The above results indicate that ANTXR1 is the cell receptor for SVA infection of porcine cells.

\section{Discussion}

Reporter-expressing viruses are useful tools in vitro and in vivo studies, such as quantitative analysis of viral replication, monitoring virus transport, screening antiviral agents, and identification of virus cell receptor, which do not require specific immunostaining of viral proteins ( $\mathrm{Li}$ et al. 2016). In this report, we described the development and characterization of three SVA reporter recombinants encoding Nluc, iLOV, and RFP. The reporter viruses showed growth kinetics comparable to the V-GD05-clone (Fig. 4e). These results indicated that an efficient reverse genetic system has been established for rescuing SVA reporter viruses. For construction of reporter viruses, the foreign genes were inserted between the $2 \mathrm{~A}$ and $2 \mathrm{~B}$ genes with the addition of a Teschovirus $2 \mathrm{~A}$ protease cleavage site at its $\mathrm{C}$-terminus. Upon the cleavage by SVA $2 \mathrm{~A}$ and Teschovirus 2A, the reporter proteins (iLOV, RFP, and Nluc) were released and detected (Fig. 3).

The SVA reporter virus expressing EGFP has been constructed using SVV-001 and KS15-01 strains in previous studies, but it was unstable in continuous three to five passages. In order to determine the size of exogenous gene that could be stably inserted into the SVA genome between 2A and $2 \mathrm{~B}$, we constructed three recombinant SVA expressing reporter proteins of different sizes. The flavoprotein improved LOV (iLOV) is a novel green fluorescent protein derived from the blue light receptor phototropin (Chapman et al. 2008). For certain applications such as fluorescent-tagged recombinant virus, the iLOV has been used as an alternative to the traditional GFP $(\sim 700 \mathrm{nt})$ for its smaller size $(\sim 300 \mathrm{nt})$ (Seago et al. 2013; van den Wollenberg et al. 2015). Recently, this method had been applied to screen antivirals in Rhinovirus-iLOV-infected HeLa cells (Han et al. 2018), where the number of green fluorescence-positive-infected cells decreased when they were treated with known flavivirus inhibitor (such as bafilomycin). As a novel engineered product, Nanoluciferase (Nluc) has been developed from a deepsea shrimp luciferase by directed evolution. Not only is the Nluc smaller ( $\sim 500 \mathrm{nt})$ than either firefly or Renilla luciferase, it is also more stable to environmental conditions as well as produces brighter and more sustained luminescence (Hall et al. 2012). In the cases of Nluc-expressing flaviviruses, the enzyme activity was quantified in a simple manner or a highthroughput assay to assess the antiviral activity of potential inhibitors in vitro (Cao et al. 2018; Pierson et al. 2017). The brilliantly red fluorescent protein TagRFP (RFP) is derived from Entacmaea quadricolor with a size of $\sim 700 \mathrm{nt}$ comparable to the one of GFP (Subach et al. 2008). It is characterized with long time fluorescence and high $\mathrm{pH}$ resistance, making it an attractive tag for protein studies. When these fluorescent and Nluc reporters with distinct properties are tagged into SVA genome, they offer particular benefits in the virus research.

After continuous passage of SVA reporter viruses, the results showed that the RFP and Nluc gene fragments were lost after three to five passages, while the iLOV was genetically stable over ten passages through cell culture using the same strategy. Comparing to the previous reports on the EGFPtagged SVA, the RFP and Nluc have the similar issue of unstability in the SVA genome under a few passage. In the 
FMDV study, a foreign gene of $300-400 \mathrm{nt}$ was considered as the maximum size for stable insertion into virus genome (Seago et al. 2013), which coincides with the current finding that the iLOV of $\sim 300 \mathrm{nt}$ was capable of being stably maintained in the SVA. The reasons for loss of foreign gene quickly during viral passage may involve more than one factor, such as the packaging limit of capsid, genome recombination, codon usage bias, and RNA structure (Song et al. 2012). Interestingly, a recent study show that the RNA-dependent RNA polymerase (RdRp) played a critical role in SVA recombination, where specific nonsynonymous mutations in the RdRp were capable of reducing recombination to occur, thereby increasing the stability of foreign gene in the virus genome (Li et al. 2019). In the future study, we are interested to investigate if it works on our SVA reporter viruses.

ANTXR1, also known as tumor endothelial marker 8 (TEM8), was first identified as the cell surface receptor of anthrax toxin (Miles et al. 2017). Unlike ANTXR2, another anthrax toxin receptor with a wide distribution in the adult tissues, ANTXR1, is abundant in the tumor cells and the vasculature of developing embryos (Cao et al. 2018; Chaudhary et al. 2012). Among 1037 cell lines in the Cancer Cell Line Encyclopedia, over $63 \%$ of cell lines exceed the expression cutoff of ANTXR1 (Miles et al. 2017). ANTXR1 has recently been identified as the cellular receptor for entry of SVV-001 into human cells. Although porcine ANTXR1 and human ANTXR1 share a close sequence homology of $97 \%$, there were R88Q and D156E mutations on the porcine ANTXR1. The two residues on the $\mathrm{N}$ terminal of ANTXR1 play an indispensable role on interacting with SVA VP proteins. Therefore, the question remains whether it is the case for porcine SVA infection. Here, we knocked out or overexpressed the porcine ANTXR1 on the ST-R cells. After SVA reporter virus infection, there was no specific fluorescence and luciferase activity detectable in the ST-R ANTXR1 KO cells infected with V-GD05-iLOV, VGD05-RFP, and V-GD05-Nluc, while overexpression of porcine ANTXR1 could increase the cellular permissivity for SVA infection. The results confirmed that ANTXR1 was the receptor for SVA GD05/2017 to invade porcine cells.

In summary, we have successfully constructed the iLOV, RFP, or Nluc tagged-SVA reporter viruses using a Chinese strain in this study. It was found that the iLOV-tagged SVA could maintain a high level of stable passage in cells. Furthermore, we examined the feasibility of SVA reporter virus as a research tool by fluorescence observation, flow cytometry, and luciferase activity. Importantly, our studies on the ANTXR1-knockout or -overexpressed ST-R cell suggested that ANTXR1 was the cellular receptor for SVA invasion of pig-derived cells. We believe that these reporter virus tools are useful for studying SVA pathogenesis and vaccine development as well as for screening novel anti-SVA agents.
Supplementary Information The online version contains supplementary material available at https://doi.org/10.1007/s00253-021-11181-6.

Acknowledgments We thank all of members at Dr. Chen's lab for their suggestions and excellent technical assistance

Authors' contributions MW carried out most of the experiments. CM and MC carried out complementary experiments. All authors analyzed the data. ZC and CM conceived and supervised the study. ZC, MW and $\mathrm{CM}$ designed the experiments and wrote the manuscript.

Funding This work was funded by the National Natural Science Foundation of China (grant no. 31772748), the National Key Research and Development Program of China (2017YFD0500104), and the Priority Academic Program Development of Jiangsu Higher Education Institutions (PAPD) to Z.C.

Data availability The recombinant SVA viruses generated in this study can be obtained from the corresponding author upon reasonable request.

\section{Declarations}

Ethics approval This article does not contain any studies with human participants or animals performed by any of the authors.

Consent to participate Not applicable.

Consent for publication Not applicable.

Conflict of interest The authors declare that they have no conflict of interest.

\section{References}

Adams MJ, Lefkowitz EJ, King AM, Bamford DH, Breitbart M, Davison AJ, Ghabrial SA, Gorbalenya AE, Knowles NJ, Krell P, Lavigne R, Prangishvili D, Sanfacon H, Siddell SG, Simmonds P, Carstens EB (2015) Ratification vote on taxonomic proposals to the international committee on taxonomy of viruses (2015). Arch Virol 160(7):18371850

Canning P, Canon A, Bates JL, Gerardy K, Linhares DC, Piñeyro PE, Schwartz KJ, Yoon KJ, Rademacher CJ, Holtkamp D, Karriker L (2016) Neonatal mortality, vesicular lesions and lameness associated with senecavirus A in a U.S. Sow Farm. Transbound Emerg Dis 63(4):373-378

Cao L, Zhang R, Liu T, Sun Z, Hu M, Sun Y, Cheng L, Guo Y, Fu S, Hu J, Li X, Yu C, Wang H, Chen H, Li X, Fry EE, Stuart DI, Qian P, Lou Z, Rao Z (2018) Seneca valley virus attachment and uncoating mediated by its receptor anthrax toxin receptor 1. Proc Natl Acad Sci U S A 115(51):13087-13092

Chapman S, Faulkner C, Kaiserli E, Garcia-Mata C, Savenkov EI, Roberts AG, Oparka KJ, Christie JM (2008) The photoreversible fluorescent protein iLOV outperforms GFP as a reporter of plant virus infection. Proc Natl Acad Sci U S A 105(50):20038-20043

Chaudhary A, Hilton Mary B, Seaman S, Haines Diana C, Stevenson S, Lemotte Peter K, Tschantz William R, Zhang Xiaoyan M, Saha S, Fleming T, St. Croix B (2012) TEM8/ANTXR1 blockade inhibits pathological angiogenesis and potentiates tumoricidal responses against multiple cancer types. Cancer Cell 21(2):212-226 
Chen Z, Yuan F, Li Y, Shang P, Schroeder R, Lechtenberg K, Henningson J, Hause B, Bai J, Rowland RRR, Clavijo A, Fang Y (2016) Construction and characterization of a full-length cDNA infectious clone of emerging porcine senecavirus A. Virology 497: $111-124$

Chen L, Zhang J, Wang M, Pan S, Mou C, Chen Z (2019) Pathogenicity of two chinese seneca valley virus (SVV) strains in pigs. Microb Pathog 136:103695

Corner SSK (2012) Seneca valley virus and vesicular lesions in a pig with idiopathic vesicular disease. J Vet Sci Technol 3(6):1-3

Donnelly MLL, Luke G, Mehrotra A, Li X, Hughes LE, Gani D, Ryan MD (2001) Analysis of the aphthovirus 2A/2B polyprotein 'cleavage' mechanism indicates not a proteolytic reaction, but a novel translational effect: a putative ribosomal 'skip'. J Gen Virol 82(Pt 5):1013-1025

Guo B, Piñeyro PE, Rademacher CJ, Zheng Y, Li G, Yuan J, Hoang H, Gauger PC, Madson DM, Schwartz KJ, Canning PE, Arruda BL, Cooper VL, Baum DH, Linhares DC, Main RG, Yoon KJ (2016) Novel senecavirus A in swine with vesicular disease, United States, July 2015. Emerg Infect Dis 22(7):1325-1327

Hales LM, Knowles NJ, Reddy PS, Xu L, Hay C, Hallenbeck PL (2008) Complete genome sequence analysis of seneca valley virus-001, a novel oncolytic picornavirus. J Gen Virol 89(Pt 5):1265-1275

Hall MP, Unch J, Binkowski BF, Valley MP, Butler BL, Wood MG, Otto P, Zimmerman K, Vidugiris G, Machleidt T, Robers MB, Benink HA, Eggers CT, Slater MR, Meisenheimer PL, Klaubert DH, Fan F, Encell LP, Wood KV (2012) Engineered luciferase reporter from a deep sea shrimp utilizing a novel imidazopyrazinone substrate. ACS Chem Biol 7(11):1848-1857

Han M, Rajput C, Hinde JL, Wu Q, Lei J, Ishikawa T, Bentley JK, Hershenson MB (2018) Construction of a recombinant rhinovirus accommodating fluorescent marker expression. Influenza Other Resp 12(6):717-727

Hause BM, Myers O, Duff J, Hesse RA (2016) Senecavirus A in Pigs, United States, 2015. Emerg Infect Dis 22(7):1323-1325

Leme RA, Oliveira TE, Alcântara BK, Headley SA, Alfieri AF, Yang M, Alfieri AA (2016) Clinical manifestations of senecavirus A infection in neonatal pigs, brazil, 2015. Emerg Infect Dis 22(7):1238-1241

Li Y, Li L-F, Yu S, Wang X, Zhang L, Yu J, Xie L, Li W, Ali R, Qiu H-J (2016) Applications of replicating-competent reporter-expressing viruses in diagnostic and molecular virology. Viruses 8(5):127

Li C, Wang H, Shi J, Yang D, Zhou G, Chang J, Cameron CE, Woodman A, Yu L (2019) Senecavirus-specific recombination assays reveal the intimate link between polymerase fidelity and RNA recombination. J Virol 93(13):e00576-e00519

Liu T, Li X, Wu M, Qin L, Chen H, Qian P (2019) Seneca valley virus 2C and $3 \mathrm{C}(\mathrm{pro})$ induce apoptosis via mitochondrion-mediated intrinsic pathway. Front Microbiol 10:1202

Miles LA, Burga LN, Gardner EE, Bostina M, Poirier JT, Rudin CM (2017) Anthrax toxin receptor 1 is the cellular receptor for seneca valley virus. J Clin Invest 127(8):2957-2967

Montiel N, Buckley A, Guo B, Kulshreshtha V, VanGeelen A, Hoang H, Rademacher C, Yoon KJ, Lager K (2016) Vesicular disease in 9week-old pigs experimentally infected with senecavirus A. Emerg Infect Dis 22(7): 1246-1248

Pasma T, Davidson S, Shaw SL (2008) Idiopathic vesicular disease in swine in Manitoba. Can Vet J 49(1):84-85
Pierson TC, Brecher M, Li Z, Liu B, Zhang J, Koetzner CA, Alifarag A, Jones SA, Lin Q, Kramer LD, Li H (2017) A conformational switch high-throughput screening assay and allosteric inhibition of the flavivirus NS2B-NS3 protease. PLoS Pathog 13(5):e1006411

Poirier JT, Reddy PS, Idamakanti N, Li SS, Stump KL, Burroughs KD, Hallenbeck PL, Rudin CM (2012) Characterization of a full-length infectious cDNA clone and a GFP reporter derivative of the oncolytic picornavirus SVV-001. J Gen Virol 93(Pt 12):2606-2613

Reed LJ, Muench H (1938) A simple method of estimating fifty percent endpoints. Am J Hygiene 27:493-497

Saporiti V, Fritzen JTT, Feronato C, Leme RA, Lobato ZIP, Alfieri AF, Alfieri AA (2017) A ten years (2007-2016) retrospective serological survey for seneca valley virus infection in major pig producing states of brazil. Vet Res Commun 41(4):317-321

Seago J, Juleff N, Moffat K, Berryman S, Christie JM, Charleston B, Jackson T (2013) An infectious recombinant foot-and-mouth disease virus expressing a fluorescent marker protein. J Gen Virol 94(Pt 7):1517-1527

Song S, Liu L, Edwards SV, Wu S (2012) Resolving conflict in eutherian mammal phylogeny using phylogenomics and the multispecies coalescent model. Proc Natl Acad Sci U S A 109(37):14942-14947

Subach OM, Gundorov IS, Yoshimura M, Subach FV, Zhang JH, Gruenwald D, Souslova EA, Chudakov DM, Verkhusha VV (2008) Conversion of red fluorescent protein into a bright blue probe. Chem Biol 15(10):1116-1124

van den Wollenberg DJM, Dautzenberg IJC, Ros W, Lipińska AD, van den Hengel SK, Hoeben RC (2015) Replicating reoviruses with a transgene replacing the codons for the head domain of the viral spike. Gene Ther 22(3):267-279

Vannucci FA, Linhares DC, Barcellos DE, Lam HC, Collins J, Marthaler D (2015) Identification and complete genome of seneca valley virus in vesicular fluid and sera of pigs affected with idiopathic vesicular disease, brazil. Transbound Emerg Dis 62(6):589-593

Venkataraman S, Reddy SP, Loo J, Idamakanti N, Hallenbeck PL, Reddy VS (2008a) Crystallization and preliminary X-ray diffraction studies of seneca valley virus-001, a new member of the picornaviridae family. Acta Crystallogr Sect F Struct Biol Cryst Commun 64(Pt 4):293-296

Venkataraman S, Reddy SP, Loo J, Idamakanti N, Hallenbeck PL, Reddy VS (2008b) Structure of seneca valley virus-001: an oncolytic picornavirus representing a new genus. Structure 16(10):1555-1561

Wang M, Chen L, Pan S, Mou C, Shi K, Chen Z (2019) Molecular evolution and characterization of novel seneca valley virus (SVV) strains in South China. Infect Genet Evol 69:1-7

Wang M, Jin S, Chen L, Chen Z (2020a) Complete genome sequencing and construction of an infectious clone of a SVA GD05/2017strain. J Yangzhou Univ 41(2):51-56

Wang J, Mou C, Wang M, Pan S, Chen Z (2020b) Transcriptome analysis of senecavirus A-infected cells: Type I interferon is a critical antiviral factor. Microb Pathog 147:104432

Wu Q, Zhao X, Chen Y, He X, Zhang G, Ma J (2016) Complete genome sequence of seneca valley virus $\mathrm{CH}-01-2015$ identified in China. Genome Announc 4(1):e01509-e01515

Publisher's note Springer Nature remains neutral with regard to jurisdictional claims in published maps and institutional affiliations. 